\title{
Inhalt von Band 142.
}

\section{Heft 1.}

Ausgegeben Im Juni 1928.

H. Bechhold und Robert Schnurmann, Die Ermittlung der Porenweite keramisober Filter durch die Systeme Luft / Flüssigkeit und Flüssigkeit / Flüssigkeit. (Mit 6 Figuren im Text).

N. Schlesinger und W. Kubasowa, Uber die Aussalzung von Äthylacetat. . . 25

$\boldsymbol{K}$. Bennewitz und $N$. Andreewa, Untersuchangen im kritischen Gebiet. III. Energiemessungen mittels Joule-Effektes. (Mit 6 Figuren im Text). .

Richard Glauner, Utber die Lösungsgeschwindigkeit des Kupfers. (Mit 13 Figuren im Text).

G. Bredig und R. Bayer, Dampfdrucke von Gemisohen.

Bü cherschau.

W. WiEx und F. Harms, Handbuch der Experimentalphysik. Band XV: K. W. F. KoBlaAcBCB, Radioaktivitat

Hass PRTTERsson, Kunstliche Verwandlung der Elemente (Zertrimmerung der Atome)

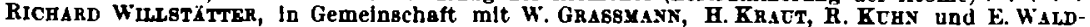
SCHMmT-LEITz, Lntersuchungen Uber Enzyme

\section{Heft 2.}

Ausgegeben Im Jull 1028.

Herbert Brandes, Elektrolytische Polarisationen aus Anlass von Kristallwachstumshemmungen. (Mit 11 Figuren im Tert) . . . . . . . . . . . .

$H$. Zocher und V. Birstein, Beiträge zur Kenntnis der Mesophasen. (Zwischenaggregatzustände.) II. Uber die gegenseitige Lagerung der Raumelemente einer Mesophese. (Mit 3 Figuren im Text) . . . . . . . . . . . . . 113

$H$. Zocher und V.Birstein, Beiträge zur Kenntnis der Mesophasen. (Zwischenaggregatzustände.) MI. Uber die wässerige Mesophase des Salvarsans - 126

E. C. Gilbert, Studien über Hydrazin: Die Geschwindigkeit der Oxydation von Hydrazin durch Ferricyanid . . . . . . . . . . . . . . . 139

M. Le Blanc und E. Möbius, Untersuchungen über Kobaltoxyde und deren Systeme mit Sauerstoff. (Mit l Figur im Text) . . . . . . . . . . . 151

\section{Heft 3.-}

Ausgegeben im Jull 1929.

H. Zocher und V. Birstein, Beiträge zur Kenntnis der Mesophasen. (Zwischenaggregatzustände.) IV. Einige weitere Fälle wässeriger Mesophasen . . . 177

H. Zocher und V. Birstein, Beiträge zur Kenntnis der Mesophasen. (Zwischenaggregatzustände.) V. Úber die Beeinflussung duroh das elektrische und magnetische Feld. (Mit 3 Figuren im Text) . . . . . . . . . . . 186

S. Kilpi, Die Einwirkung von Chlorwasserstoff auf Alkohol. VI. Geschwindigkeitskoeffizienten . . . . . . . . . . . . . 195

N. A. Puschin und T. Pinter, Viskosität binärer Systeme mit Guajakol als Komponente. (Mit 10 Figuren im Text) . . . . . . . . . . . . . . 211

Adolf Pabst, Röntgenuntersuchung über die Bildung von Zinksilicaten . . . . 227

D. Talmud, Uber die Orientierung der Moleküle an fester Grenzfläche und über die Reichweite der orientierenden Kräfte. (Mit 1 Figur im Text.) Vorläufige Mitteilung!.

Bü cherschau.

W. WIEx und F. HABYs, Handbuch der Experimentalphysik. Bd. VII/2: Strukturbestimmung mit Röntgeninterferenzen von HEINRICB OTT. - Gittertheorje der festen Körper von KaRL

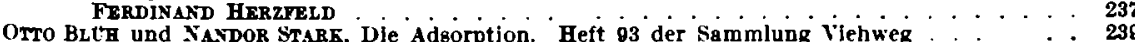

Wilhely $\mathbf{H}$. Westphal, Physik, ein Lehrbuch für Studierende an den Enivergităten und Technischen Hochschulen . . . . . . . . . . . . . . . . . . . . . . 


\section{Heft 4.}

Auvgegeben im Juli 1929.

L. Tronstad, Optische Untersuchungen an elektrochemisch aktivierten und passivierten Metallspiegeln. (Mit 10 Figuren im Text) . . . . . . . . . . 241

P. Rehbinder und Fr. L. Krajuschkina, Benetzungewärmen von Pulvern mit Lösungen grenzflächenaktiver Stoffe und Adsorptionswärmen in Lösungen. (Wärmeeffekte bei Bildung disperser Systeme. II) . . . . . . 282

Herbert Pollatschek, Die Bestimmung der an der Grenze fest/flüssig während der Kristallisation unterkühlter Schmelzen berrschenden Temperatur. (Mit 8 Figuren im Text).

$M$. Gehrke und H. H. Willrath, Uber Gleichgewichte zwischen Oxysäuren und ihren Anhydroformen. (Mit 1 Figur im Text) . . . . . . . . . . . 301

Anneliese Niethammer, Permeabilitätastudien an Pflanzenzellen im Zusammenhange mit der Quecksilberbeizung der Samen . . . . . . . . . . 309

A u fruf für Bewerber um ein Stipendium aus der vaN'T HoF -Stiftung zur Unterstuitzung von Forschern auf dem Gebiete der reinen oder angewandten Chemie 320

\section{Heft $\overline{\text { o. }}$}

Ausgegeben im August 1929.

E. $\boldsymbol{X}$. Anderson und J. A. Froemke, Die Kernbildung bei der Kondensation von Dämpfen in nicht ionisierter staubfreier Luft. (Mit 8 Figuren im Text) 321

$E$. Lange und $E$. $O$. Rounsefell, Bemerkungen zur adiabatischen Calorimetrie. I. Bei Temperaturänderungen in der Grössenordnung von $1^{\circ}$. (Mit 4 Figuren im Text) . . . . . . . . . . . . . .

Erich Schröer, Untersuchungen über den kritischen Zustand IV. Kritischer Zustand der Athylätherlösungen. (Mit 9 Figuren im Text) . . . . . 365

\section{Bücherschau.}

Jkrome Almxander, Collold Chemistry, Theoretical and Applied. Bd. II. Biology and Medizine 391

ARNOLD BERLINER, Lehrbuch der Phygik in elementarer Darstellung. . . . . . . . . . 392

Michrle Grod, Elementi die Chimica Organlca con Trattazione Particolare della Chimica di Guerra (Elemente der organischen Chemle mit besonderer Berlckeichtigung der Krlegs-

Prof. Dr. Wo. Ostwisd, Gallerten und Gele in silgemeln kolloidchemischer, biologischer und technischer Hinsicht.

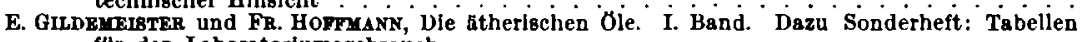
filir den Laboratoriumsgebrauch .

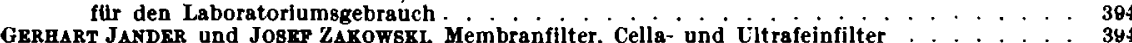

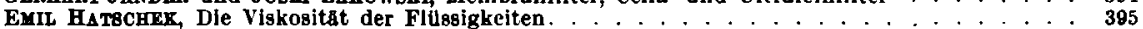

Ergebnisse der exakten Naturwigsenschaften. Band I. . . . . . . . . . . . . . . . . . . . . . 396

OTTo SACRUR t, Lehrbuch der Thermochemie und Thermodynamik . . . . . . . . . . . . . 396

KARL JrLLnNeK, Lehrbuch der phyikalischen Chemle. 2. Band . . . . . . . . . . . . . . . . . 397

F. F. P. BIBACRE, Prakt/sche Infiniteslmalrechnung . . . . . . . . . . . . . . . . . . . . . . . 397

T. P. Hudrch, Catalytic Processes in applled chemistry . . . . . . . . . . . . . . . 398

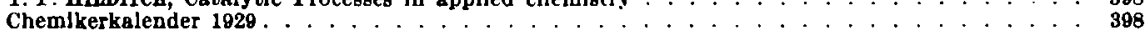

Eingegangene Bücher. . . . . . . . . . . . . . . . . . . . . . . . . . 399

\section{Heft 6.}

Ausgegeben im August 1029.

A. Magnus, Theorie der Gasadsorption. (Mit 3 Figuren am Text) . . . . . . 401 Witali Heller, Uber die Möglichkeit, kinetische Prozesse in heterogenen Systemen bei einheitlichen Rübrungebedingungen zu charakterisieren. (Mit 4 Figuren im Text) . . . . . . . . . . . . . . . . . .

$I, N$. Stranski, Zur Theorie der isomorphen Fortwachsung (orientierter Aussoheidung ron Ionenkristallen aufeinander. (Mit 1 Figur im Text). . . 453

I. N. Stranski und $K$. Kuleliew, Beitrag zur isomorphen Fortwachsung von Ionenkristallen aufeinender ..................467

Bücherschau.

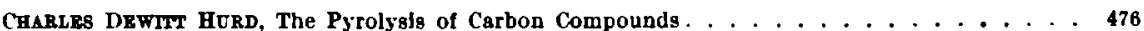

JoHN E. TEkPLe, The industrial developement of the Searles lake brines . . . . . . . . 470 